\title{
Impacto de diferentes soluções eletrolíticas orais sobre a glicemia e os equilíbrios hídrico, eletrolítico e ácido-base de bezerros neonatos sadios
}

[Effects of different oral electrolyte solutions on the glycemia and water, electrolyte, and acid-base balances of healthy neonatal calves]

\author{
G.C. Bregadioli, F.C. Pinto, J.M. Curti, S.L.O. Camilo, K.K.M.C. Flaiban, J.A.N. Lisbôa*
}

Universidade Estadual de Londrina - Londrina, PR

\begin{abstract}
RESUMO
Este trabalho objetivou estudar os efeitos de quatro soluções eletrolíticas orais (SEO) comerciais, de uma SEO não comercial (SEO UEL) e da solução de ringer com lactato (SRL), sobre a glicemia e os equilíbrios hídrico, eletrolítico e ácido-base de bezerros neonatos sadios. Utilizaram-se seis bezerros, os quais receberam todas as seis SEO, uma por vez, e cada tratamento foi realizado no período de um dia. Amostras de sangue venoso foram coletadas, em diferentes momentos do dia de tratamento, para a determinação da proteína plasmática total, glicemia e hemogasometria. Valores de $\mathrm{SID}_{3}$ e variação do volume plasmático foram calculados. A análise de variância de medidas repetidas foi empregada para comparação entre os momentos e as soluções. As SEO não provocaram alterações de magnitude alta ou prolongadas nos equilíbrios hídrico, eletrolítico e ácido-base dos bezerros. Pode-se concluir que todas as SEO, à exceção da SEO A, originaram expansão da volemia. As SEO B e C originaram efeito alcalinizante, enquanto a D afetou a glicemia, e a SRL aumentou a cloremia. A SEO UEL não produziu nenhum efeito marcante além da expansão do volume plasmático.
\end{abstract}

Palavras-chave: hidratação enteral, bovinos, terapia com fluidos

\begin{abstract}
This work aimed to study the effects of four commercially available oral electrolyte solutions (OES), of a non-commercial OES (OES UEL), and of the lactated Ringer's solution (LRS) administered orally, on the glycemia and water, electrolyte, and acid base balance of healthy neonatal calves. Six calves were used, all of them received the six OES, one at a time, and each treatment was performed within one day. Venous blood samples were taken, in different moments in the treatment day, for determination of total plasma protein, glycemia and blood gas analysis. Values of $\mathrm{SID}_{3}$ and plasma volume variation were calculated. Repeated measures ANOVA was used for comparison between moments and solutions. The OES studied did not induced changes of high or prolonged magnitude in the water, electrolyte, and acid base balances of the calvesIn conclusion, all OES, with the exception to OES A, led to expansion of the plasma volume. The OES B and C had an alkalizing effect while D affected glycemia and LRS increased chloremia. The OES UEL did not produce any marked effect, besides the expansion of plasma volume.
\end{abstract}

Keywords: enteral hydration, cattle, fluid therapy

\section{INTRODUÇÃO}

No século XX, o uso de soluções eletrolíticas orais (SEO) evitou a morte de milhares de pessoas, notadamente crianças, com desidratação e diarreia, principalmente nos países em desenvolvimento. A partir dos resultados positivos obtidos com o uso de SEO em crianças, a terapia com fluidos por via oral passou a ser utilizada na medicina veterinária e se tornou o tratamento de escolha das diarreias neonatais em bezerros (Smith, 2009).

Recebido em 15 de agosto de 2017

Aceito em 3 de novembro de 2017

*Autor para correspondência (corresponding author)

E-mail: janlisboa@uel.br 
Para que a hidratação oral seja capaz de corrigir, efetivamente, a desidratação e os desequilíbrios eletrolíticos, é recomendável que as SEO possuam composição com características desejáveis. $\mathrm{O} \mathrm{Na}^{+}$deve estar presente em quantidade suficiente para suprir o déficit e restabelecer o volume do espaço fluido extracelular. Dois ou mais agentes que facilitem a absorção de $\mathrm{Na}^{+}$e de água pelo intestino, tais como glicose, acetato, propionato ou glicina, devem ser veiculados. $\mathrm{O} \mathrm{K}^{+}$deve estar contido para repor as perdas. Um agente alcalinizante, como o acetato ou o bicarbonato, deve estar presente, com a finalidade de corrigir ou amenizar a acidose metabólica. E, finalmente, a glicose é necessária para evitar ou reverter o balanço energético negativo (Naylor, 1990; Constable, 2003).

Na América do Norte, a reposição oral de fluidos e eletrólitos é um procedimento consolidado e praticado desde a década de 70 no tratamento de bezerros diarreicos desidratados, existindo, pelo menos, 20 alternativas comerciais disponíveis (Naylor, 1990; Smith e Berchtold, 2014). No Brasil, ao contrário, o uso de SEO não era comum, porém, nos últimos anos, a prática da hidratação oral de bezerros vem se expandindo. Atualmente existem cinco produtos disponíveis no mercado para o preparo de SEO, o que significa grande avanço terapêutico.

A hipótese que sustenta o presente estudo é a de que SEO com composições diferentes determinam impactos distintos sobre a água e os eletrólitos do organismo. Com base nisso, tornase importante conhecer os efeitos que cada SEO provoca ao ser ingerida. Poucos trabalhos testaram as SEO disponíveis comercialmente no país com o intuito de avaliar a eficiência e o benefício que elas podem trazer quando utilizadas. A hidratação com SEO preparada com Enerlyte Plus ${ }^{\circledR}$ gerou alcalinização em bezerros diarreicos e foi eficaz para reverter a desidratação (Pagliosa et al., 2013). Esse foi o único estudo realizado no Brasil. Resultados com a ingestão de SEO preparada com Glutellac ${ }^{\circledR}$ comprovaram que não houve efeito alcalinizante em bezerros sadios (Bachmann et al., 2009). Constable et al. (2009) testaram o Rehydion ${ }^{\circledR} \mathrm{Gel}$ em bezerros sadios, porém, em vez de prepararem a SEO, administraram o produto diluído no leite, o que provocou alcalinização sistêmica leve e pouca interferência com o volume plasmático.

Os autores desconhecem informações científicas sobre os efeitos da ingestão das demais SEO preparadas com os produtos disponíveis, e não há estudo que compare os efeitos delas. O objetivo deste trabalho foi comparar os efeitos de SEO, comerciais e não comerciais, sobre a glicemia e os equilíbrios hidroeletrolítico e ácido-base de bezerros neonatos sadios. A hipótese que sustenta o estudo é a de que as SEO com diferentes composições provoquem efeitos distintos sobre a glicemia e os equilíbrios hídrico, eletrolítico e ácido-base de bezerros neonatos sadios.

\section{MATERIAL E MÉTODOS}

O projeto foi aprovado pela Comissão de Ética no Uso de Animais da Universidade Estadual de Londrina (UEL), sob protocolo Ceua/UEL $n^{\circ}$ 30823.2014.52. Participaram do estudo seis bezerros sadios da raça Holandesa preta e branca (HPB), machos, com dois a três dias de idade. Na chegada ao Hospital Veterinário da UEL, os bezerros receberam aplicação única de ferro dextrânico injetável $\left(\right.$ Ferrodex $^{\circledR}$; Fabiani Saúde Animal), por via intramuscular, na dose de $4 \mathrm{~mL}$, e nova desinfecção do umbigo foi realizada com tintura de iodo $10 \%$. Durante todo o período experimental, os bezerros foram mantidos em baias individuais, previamente desinfetadas e cobertas com cama de cepilho. A limpeza da baia era realizada diariamente com a retirada de fezes. A troca completa da cama era efetuada a cada três dias.

A alimentação dos bezerros consistiu na oferta diária de sucedâneo lácteo (SL) Milk Sweet ${ }^{\circledR}$ (Nutron Alimentos Ltda.), preparado de acordo com a recomendação do fabricante, em volume correspondente a $12 \%$ do peso corpóreo (PC), dividido em duas vezes ao dia, nos horários das oito horas (6\% PC) e das $18 \mathrm{~h} 30$ (6\% PC), e fornecido por meio de mamadeira. A água foi mantida com livre acesso para os bezerros durante todo o dia, salvo apenas nos primeiros 30 minutos após a mamada, até que diminuísse o efeito de sucção do leite. A partir do quinto dia de vida, foi ofertada ração pré-inicial comercial e feno de coast-cross ad libitum. Os bezerros permaneceram em período de adaptação por $12 \mathrm{a}$ 13 dias. 
As soluções eletrolíticas orais (SEO) testadas foram preparadas com quatro produtos comerciais disponíveis no mercado brasileiro: Rehydion ${ }^{\circledR}$ Gel (Ceva Saúde Animal Ltda.) (SEO A), Glutellac ${ }^{\circledR}$ (Bayer Saúde Animal) (SEO B), Enerlyte Plus ${ }^{\circledR}$ (Virbac do Brasil Indústria e Comércio Ltda.) (SEO C) e Nutronlyt ${ }^{\circledR}$ (Nutron Alimentos Ltda.) (SEO D), todas com indicação para promover a hidratação oral de bezerros neonatos; além de uma solução eletrolítica não comercial (SEO UEL), utilizada na rotina clínica do Hospital Veterinário da Universidade Estadual de Londrina. A solução de ringer com lactato (SRL) (Laboratório Sanobiol Ltda.) comercial foi empregada por via oral, servindo como controle por não conter glicose. A composição e a osmolaridade de cada SEO estão expostas na Tab. 1.

Todas as soluções foram preparadas de acordo com a orientação do fabricante, imediatamente antes de serem administradas aos bezerros, utilizando-se água morna $\left(38^{\circ} \mathrm{C}\right)$. A SEO UEL foi preparada dissolvendo-se a mistura dos ingredientes previamente pesados em dois litros de água. Para tanto, foram empregados os seguintes compostos puros para análise (Synth; Labsynth): $\mathrm{NaCl}, \mathrm{KCl}$, acetato de sódio trihidratado e D-glicose anidra.

Tabela 1. Composição das soluções eletrolíticas orais (SEO) empregadas no estudo

\begin{tabular}{lcccccc}
\multicolumn{1}{c}{ Composição } & SEO-UEL & SEO A $^{1}$ & SEO B $^{2}$ & SEO C $^{3}$ & SEO D $^{4}$ & SRL $^{5}$ \\
\hline $\mathrm{Na}^{+}(\mathrm{mEq} / \mathrm{L})$ & 100 & 120 & 100,1 & 96,8 & 124,6 & 130 \\
$\mathrm{~K}^{+}(\mathrm{mEq} / \mathrm{L})$ & 20,6 & 22,6 & 18,5 & 16,1 & 27,7 & 4 \\
$\mathrm{Cl}^{-}(\mathrm{mEq} / \mathrm{L})$ & 86 & 82 & 60,7 & 69,4 & 96,8 & 109 \\
$\mathrm{HCO}_{3}{ }^{-}(\mathrm{mEq} / \mathrm{L})$ & -- & -- & -- & 20,9 & 55,5 & -- \\
Acetato $(\mathrm{mEq} / \mathrm{L})$ & $35^{*}$ & $60^{*}$ & $58^{*}$ & -- & -- & -- \\
Glicose $(\mathrm{mmol} / \mathrm{L})$ & 100 & 23,3 & 46 & $0^{* *}$ & 109 & -- \\
SID efetiva $(\mathrm{mEq} / \mathrm{L})^{6}$ & 34,6 & 60,6 & 58 & 43,5 & 55,5 & 28 \\
Osmolaridade (mOsm/L) & 326 & 305,6 & 283 & 203 & 402,7 & 275 \\
$\mathrm{Na}: \mathrm{Glicose}$ & $1: 1$ & $5,1: 1$ & $2,1: 1$ & -- & $1,1: 1$ & -- \\
$\mathrm{Na}: \mathrm{Cl}$ & $1,1: 1$ & $1,4: 1$ & $1,6: 1$ & $1,4: 1$ & $1,3: 1$ & $1,2: 1$ \\
$\mathrm{pH}$ & 7,502 & 7,021 & 6,232 & 7,952 & 6,606 & 6,415 \\
\hline
\end{tabular}

${ }^{1}$ Rehydion ${ }^{\circledR}$ Gel; ${ }^{2}$ Glutellac ${ }^{\circledR} ;{ }^{3}$ Enerlyte Plus ${ }^{\circledR} ;{ }^{4}$ Nutronlyt ${ }^{\circledR} ;{ }^{5}$ solução de ringer com lactato; ${ }^{6}$ SID: diferença de íons fortes (strong ion difference) calculada por $\left(\left[\mathrm{Na}^{+}\right]+\left[\mathrm{K}^{+}\right]\right)-\left[\mathrm{Cl}^{-}\right] ;{ }^{*}$ valor calculado; ${ }^{* *}$ contém lactose.

Quando os bezerros atingiram 15 dias de vida e $44,8 \pm 3,6 \mathrm{~kg}$ de PC, estavam aptos para o início da fase experimental. Foi adotado o delineamento experimental cross-over, no qual todos os bezerros receberam todos os tratamentos (as seis SEO), cada um por vez. A ordem de realização de cada tratamento foi estabelecida por sorteio. Cada tratamento foi realizado ao longo de um dia (24 horas), e o próximo tratamento somente foi administrado três a quatro dias após, período suficiente para que se restabelecesse a homeostasia. Ao término do experimento, os bezerros tinham 33 dias de idade e pesavam $48,5 \pm 4,4 \mathrm{~kg}$.

Nos dias de tratamento, dois litros da SEO foram administrados, por meio de mamadeira, em dois momentos: às $11 \mathrm{~h} 30$ e às $15 \mathrm{~h}$, totalizando quatro litros no dia. $\mathrm{O}$ fornecimento de sucedâneo lácteo foi mantido como de costume, bem como a água, a ração e o feno durante todo o dia. Os volumes recebidos das SEO relativos ao $\mathrm{PC}$ foram homogêneos entre os tratamentos, variando de 85,7 a $89,4 \mathrm{~mL} / \mathrm{kg}$ em média. O acompanhamento dos animais para observação de comportamento, apetite e atitudes foi contínuo durante todo o dia de cada tratamento e no dia seguinte. A aceitação de cada uma das SEO estudadas foi avaliada subjetivamente por um observador específico, que acompanhou todas as mamadas e que desconhecia o tipo de SEO que estava sendo administrada.

Para a coleta das amostras de sangue venoso, a veia jugular foi mantida cateterizada (cateter $16 \mathrm{G}$ ) durante todo o dia do estudo, e o cateter permaneceu vedado com um adaptador luer de fechamento (PRN). As amostras de sangue venoso foram coletadas com auxílio de seringa e agulha, em seis momentos predeterminados no 
dia da realização de cada tratamento: às oito horas (antes da primeira mamada; hora 0); às $11 \mathrm{~h} 30$ (antes da primeira administração da solução oral; hora 3,5); às 15 h (antes da segunda administração da solução oral; hora 7); às 18 h30 (antes da mamada da tarde; hora 10,5); às $22 \mathrm{~h}$ (hora 14) e às oito horas do dia seguinte (hora 24). Para avaliar o efeito da ingestão do SL, no dia anterior ao do primeiro tratamento, foi realizado o mesmo procedimento de coleta de amostras.

As amostras de sangue coletadas foram acondicionadas em frascos a vácuo contendo anticoagulante EDTA, sem fluoreto de sódio, para a determinação da concentração de proteínas plasmáticas totais (PPT), e com fluoreto de sódio, para a determinação da glicemia. O plasma fluoretado foi obtido por centrifugação da amostra em até, no máximo, 10 minutos após a coleta e conservado por congelação ( $20^{\circ} \mathrm{C}$ negativos) até o momento das análises.

Para a hemogasometria, amostras de sangue foram coletadas, sob condição anaeróbica, empregando-se seringas plásticas de $3 \mathrm{~mL}$, contendo cerca de $0,08 \mathrm{~mL}$ (400UI) de heparina sódica $\quad\left(\right.$ Hemofol $^{\circledR} ; \quad$ Cristália) como anticoagulante, acopladas a agulhas hipodérmicas $21 \mathrm{G}(0,8 \times 30 \mathrm{~mm})$. As análises foram realizadas logo após a coleta.

Os exames laboratoriais consistiram na determinação da concentração de PPT por refratometria e da concentração plasmática de glicose, mediante a utilização do método colorimétrico (Dimension; Siemens). O exame hemogasométrico consistiu nas determinações sanguíneas de $\mathrm{pH}, \mathrm{pCO}_{2}, \mathrm{HCO}_{3}{ }^{-}, \mathrm{BE}, \mathrm{Na}^{+}, \mathrm{K}^{+}$e $\mathrm{Cl}^{-}$(Omni C; Roche).

As seguintes variáveis foram calculadas empregando-se as respectivas fórmulas:

a) diferença de íons fortes - Strong Ion Difference (SID): $\mathrm{SID}_{3}=\left(\mathrm{Na}^{+}+\mathrm{K}^{+}\right)-\left(\mathrm{Cl}^{-}\right)$;

b) variação do volume plasmático $(\mathrm{VVP})$ : $\mathrm{VVP}=$ $\left(\mathrm{PPT}_{0}-\mathrm{PPT}_{\mathrm{i}}\right) \times 100 / \mathrm{PPT}_{\mathrm{i}}$; sendo $\mathrm{PPT}_{0}$ e $\mathrm{PPT}_{\mathrm{i}}$ a concentração de proteínas plasmáticas na hora 0 e no momento i, respectivamente (Van Beaumont et al., 1972).

A análise de variância de medidas repetidas bifatorial foi empregada para testar o efeito do fator tempo (diferentes momentos antes e após a administração da SEO), do fator tratamento (diferentes SEO) e da interação entre os dois fatores. Para verificar o efeito isolado da ingestão do sucedâneo de leite, empregou-se a análise de variância de medidas repetidas unifatorial. O teste de Tukey foi empregado para comparação entre as médias. Admitiu-se a probabilidade de erro de 5\% (SigmaPlot for Windows 13.0; Systat Software Inc.).

\section{RESULTADOS}

Todas as SEO utilizadas no estudo foram bem aceitas pelos bezerros, que as mamaram sempre avidamente e com sucção vigorosa, sem demonstrarem preferência. Os diferentes tipos de SEO utilizados provocaram efeitos sobre os valores de $\mathrm{pH}(\mathrm{P}=0,002), \mathrm{HCO}_{3}{ }^{-}(\mathrm{P}<0,001), \mathrm{BE}$ ( $\mathrm{P}<0,001), \mathrm{Cl}^{-}(\mathrm{P}=0,030), \mathrm{SID}_{3}(\mathrm{P}=0,013), \mathrm{VVP}$ $(\mathrm{P}=0,080)$ e glicose $(\mathrm{P}<0,001)$. As SEO A, B e C geraram $\mathrm{pH}$ sanguíneo mais elevado do que a SRL, e as quatro soluções preparadas com produtos comerciais (SEO A, B, C e D) produziram valores mais altos de $\mathrm{HCO}_{3}{ }^{-}$e de $\mathrm{BE}$ do que a SRL. A SEO UEL gerou valores intermediários para essas variáveis, os quais não diferiram dos observados com a ingestão da SRL (Fig. 1). A cloremia observada após a ingestão das SEO comerciais apresentou-se, em geral, menor do que a observada após a ingestão da SRL. Os valores de $\mathrm{Cl}^{-}$foram intermediários com a ingestão da SEO UEL. No caso da $\mathrm{SID}_{3}$, os valores após a ingestão da SEO C foram maiores do que os observados com a ingestão da SRL e da SEO UEL. A VVP diferiu, marcadamente, entre as SEO na hora 14, e os valores foram mais reduzidos após a ingestão da SEO A e da SRL, comparando-se à ingestão da SEO D. Os valores de glicose foram maiores com a ingestão da SEO D, e isso é destacado nas horas 7 e 10,5 (Fig. 2). Os efeitos do fator tempo foram confirmados para o $\mathrm{pH}(\mathrm{P}=0,004)$, a $\mathrm{pCO}_{2}(\mathrm{P}=0,04),{\mathrm{o} \mathrm{HCO}_{3}}^{-}$ $(\mathrm{P}<0,001)$, o BE $(\mathrm{P}<0,001), \mathrm{o} \mathrm{Na}^{+}(\mathrm{P}<0,001)$, o $\mathrm{K}^{+}(\mathrm{P}<0,049)$, o Cl$^{-}(\mathrm{P}<0,001)$, a $\mathrm{SID}_{3}(\mathrm{P}=0,004)$, a VVP $(\mathrm{P}<0,001)$ e a glicose $(\mathrm{P}<0,001)$. As variações do $\mathrm{pH}$ e da $\mathrm{pCO}_{2}$ foram observadas somente com a ingestão das SEO B e C, respectivamente. Incrementos nos valores de $\mathrm{HCO}_{3}^{-}$e de $\mathrm{BE}$ foram gerados com administração das SEO B e C e foram marcantes na hora 10,5 (Fig. 1). As SEO UEL e D não provocaram efeitos sobre os eletrólitos plasmáticos. $\mathrm{O} \mathrm{Na}^{+}$se elevou com as $\mathrm{SEO} A, \mathrm{C}$ e 
SRL, a potassemia não se modificou com as ingestões das SEO, e a cloremia se elevou apenas com a ingestão da SRL, e isso foi notável na hora
10,5. A $\mathrm{SID}_{3}$ se elevou na hora 10,5 com as SEO $\mathrm{B}$ e C, exclusivamente (Fig. 2).
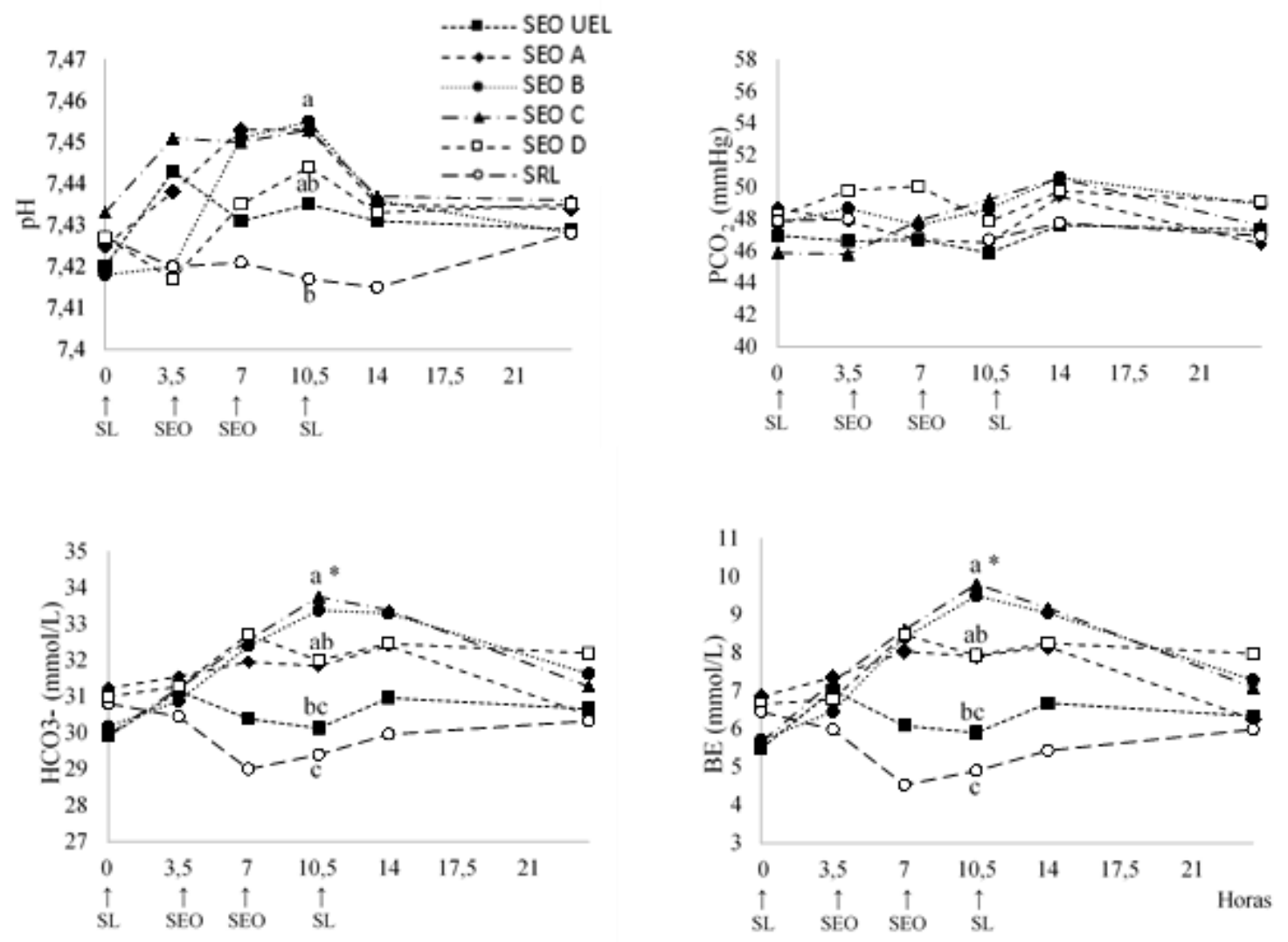

Figura 1. Variação do $\mathrm{pH}$, da $\mathrm{pCO}_{2}$, da concentração de bicarbonato $\left(\mathrm{HCO}_{3}{ }^{-}\right)$e do excesso de bases $(\mathrm{BE})$ de bezerros neonatos sadios que receberam soluções eletrolíticas orais (SEO) com diferentes composições e a solução de ringer com lactato (SRL), mamadas em volume de $2 \mathrm{~L}$ nas horas 3,5 e 7, entre as refeições de sucedâneo lácteo (SL). Letras minúsculas indicam diferença entre as SEO $(\mathrm{P}<0,05)$ * indica diferença comparada com a hora $0(\mathrm{P}<0,05)$, somente para as SEO B e C.

Como observações marcantes nas horas 10,5 e 14 , os valores da VVP aumentaram. Isso ocorreu com a ingestão de todas as SEO estudadas, excetuando-se a SEO A. De forma geral, a glicemia exibiu variações que refletem a influência da ingestão do SL, com valores mais elevados nas horas 3,5 e 14. A ingestão da SEO $\mathrm{D}$ provocou feito distinto na glicemia, e os valores mantiveram-se elevados ao longo de todo o dia (entre as horas 3,5 e 14) (Fig. 2). 
Bregadioli...
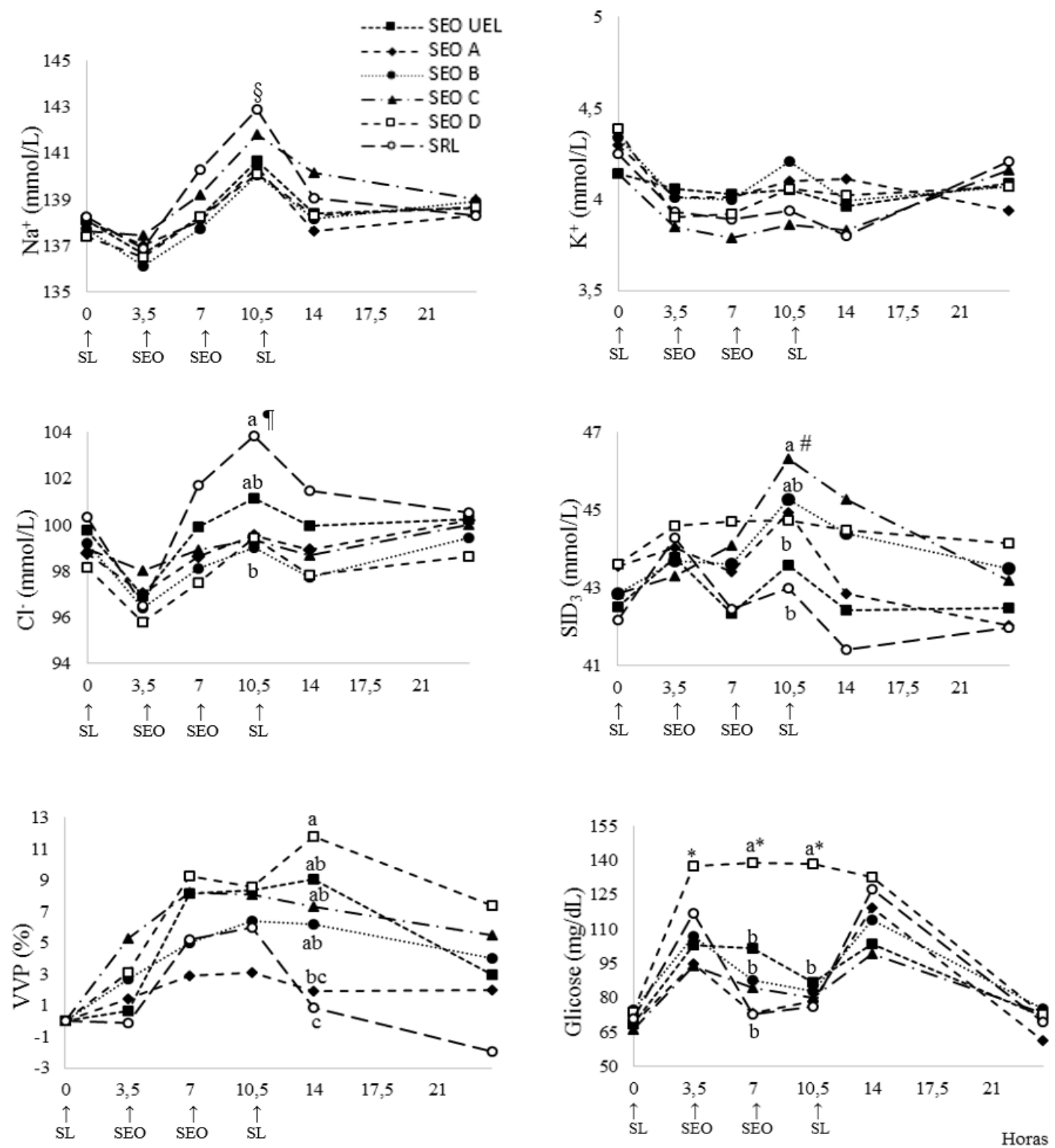

Figura 2. Variação das concentrações de $\mathrm{Na}^{+}, \mathrm{K}^{+}, \mathrm{Cl}^{-}$, da diferença de íons fortes $\left(\mathrm{SID}_{3}\right)$, da VVP e da glicose de bezerros neonatos sadios que receberam soluções eletrolíticas orais (SEO) com diferentes composições e a solução de ringer com lactato (SRL), mamadas em volume de $2 \mathrm{~L}$ nas horas 3,5 e 7, entre as refeições de sucedâneo lácteo (SL). § indica diferença comparada com a hora $0(\mathrm{P}<0,05)$, somente para as SEO A, C e SRL. Letras minúsculas indicam diferença entre as SEO $(\mathrm{P}<0,05)$. * indica diferença comparada com a hora $0(\mathrm{P}<0,05)$, somente para a SEO D. II indica diferença comparada com a hora 0 $(\mathrm{P}<0,05)$, somente para a SRL. \# indica diferença comparada com a hora $0(\mathrm{P}<0,05)$, somente para as SEO B e C.

Por fim, o efeito isolado da ingestão do SL foi confirmado para as variáveis $\mathrm{pH}(\mathrm{P}=0,04)$, $\mathrm{HCO}_{3}^{-}(\mathrm{P}=0,019), \quad \mathrm{BE} \quad(\mathrm{P}=0,007)$ e glicose
$(\mathrm{P}<0,001)$. As demais variáveis estudadas mantiveram-se sem modificações ao longo do dia. 


\section{DISCUSSÃO}

As SEO utilizadas neste estudo diferem, principalmente, em relação às concentrações de $\mathrm{Cl}^{-}$e de glicose, aos valores da SID efetiva e da osmolaridade e aos tipos e concentrações dos agentes alcalinizantes. Essas diferenças em suas composições foram, de maneira geral, responsáveis pelas modificações observadas em algumas variáveis. A ingestão do SL, por si só, não provocou interferência nos resultados obtidos, porque, com exceção da glicemia, cujas variações caracterizaram os picos glicêmicos pós-prandiais, as variáveis estudadas não se modificaram. As variações observadas para o $\mathrm{pH}, \mathrm{HCO}_{3}{ }^{-}$e $\mathrm{BE}$ foram discretas ao longo do dia.

Segundo Constable et al. (2001), Constable (2003) e Smith (2009), as SEO com composições adequadas devem apresentar concentrações de $\mathrm{Na}^{+}$entre 90 e $130 \mathrm{mEq} / \mathrm{L}$, de $\mathrm{K}^{+}$entre 10 e $20 \mathrm{mEq} / \mathrm{L}$, de $\mathrm{Cl}^{-}$entre 40 e $80 \mathrm{mEq} / \mathrm{L}$, e de bases metabolizáveis $\left(\mathrm{HCO}_{3}{ }^{-}\right.$ou acetato $\left.{ }^{-}\right)$entre $40 \mathrm{e}$ $80 \mathrm{mEq} / \mathrm{L}$; SID efetiva entre 60 e $80 \mathrm{mEq} / \mathrm{L}$, relação sódio:glicose entre $1: 1$ e $3: 1$, e osmolaridade variando entre 300 e $600 \mathrm{mOsm} / \mathrm{L}$. Com base nesses parâmetros, pode-se dizer que as SEO preparadas com os produtos comerciais brasileiros (A, B, C e D) possuem composições apropriadas para uso terapêutico em bezerros.

A julgar pela variação observada nos valores da VVP, pode-se afirmar que todas as soluções estudadas provocaram expansão do volume plasmático, excetuando-se a SEO A. Isso comprova que foram absorvidas com facilidade e que a hora 10,5 é o momento mais marcante para a interpretação dos resultados porque reflete o somatório dos efeitos das duas ingestões de SEO. A ausência de diferença entre as SEO nesse momento indica que elas se equivalem quanto à velocidade com que são absorvidas e quanto à capacidade de produzir hemodiluição. Na hora 14, momento que reflete os efeitos somados das duas ingestões de SEO e da segunda refeição de SL, fica evidente que a expansão do volume plasmático foi mais duradoura com a ingestão da SEO D em comparação com a da SEO A e da SRL (Fig. 1). A magnitude da VVP observada no presente estudo é coerente com os resultados obtidos em outros estudos, nos quais bezerros sadios experimentaram expansão do volume plasmático após ingerirem SEO (Nouri e Constable, 2006; Bachmann et al., 2009; 2012).
No caso particular da SEO A, a ausência de VVP comprovou que a sua ingestão não foi acompanhada por hemodiluição consistente, efeito que a distingue das demais. Uma característica que a diferencia das outras SEO estudadas é a concentração mais reduzida de glicose e a relação Na:glicose elevada. Uma vez que a absorção intestinal de $\mathrm{Na}^{+}$e de água é facilitada pela glicose (Naylor, 1990; Smith e Berchtold, 2014), pode-se levantar a hipótese de que a sua absorção seja menos eficiente. Contudo, essa explicação não deve ser aceita como verdadeira, porque a SRL não possui glicose em sua composição e gerou aumento do volume plasmático, assim como as demais SEO estudadas. Quando administrada anteriormente em bezerros sadios, a VVP produzida pela SEO A ficou comprovada, porém foi discreta (Constable et al., 2009). Cabe mencionar que, ao contrário do presente estudo, a SEO A foi administrada diluída no leite e não em água, o que dificulta a comparação entre os resultados.

Pode-se assumir que a ingestão das SEO incluídas no estudo não provocou alterações no equilíbrio eletrolítico dos bezerros, pois as variações de $\mathrm{Na}^{+}$, de $\mathrm{K}^{+}$e de $\mathrm{Cl}^{-}$foram discretas. As principais modificações ocorreram após a ingestão da SRL e se caracterizaram pela elevação da cloremia. Apesar de ser uma solução eletrolítica indicada para uso intravenoso, foi utilizada, no presente trabalho, por via oral. O fato de não veicular glicose foi o fator decisivo para a escolha dela como solução controle, considerando-se o impacto sobre a glicemia. Além disso, a SRL apresenta SID efetiva de $28 \mathrm{mEq} / \mathrm{L}$, menor do que todas as SEO estudadas (Tab. 1) e abaixo do que é recomendado, o que reduz o seu potencial alcalinizante. Quando comparada às outras SEO, a SRL possui concentração de $\mathrm{K}^{+}$menor e concentrações de $\mathrm{Na}^{+}$e de $\mathrm{Cl}^{-}$maiores, e isso pode explicar a cloremia mais elevada nos bezerros após a sua ingestão. Cosenza et al. (2013) demonstraram que a SRL, quando administrada por via intravenosa, em volume equivalente a $10 \%$ do PC, causou hemodiluição, mas não interferiu nos equilíbrios eletrolítico e ácido-base de bezerros sadios. Isso reforça os resultados obtidos com a administração oral, porque a elevação da cloremia foi discreta e não caracterizou desequilíbrio iatrogênico. 
Quanto à $\mathrm{SID}_{3}$ plasmática, variável que reflete a relação entre os eletrólitos e notadamente entre o $\mathrm{Na}^{+}$e o $\mathrm{Cl}^{-}$, as SEO B e C foram as únicas que provocaram aumento ao longo do dia. Na hora 10,5, ficou evidente que a SEO $\mathrm{C}$ provocou maior elevação do valor da $\mathrm{SID}_{3}$ quando comparada à SEO UEL e à SRL (Fig. 2). Apesar de os valores distintos das SID efetivas das SEO não explicarem as diferenças observadas, deve-se destacar que as SEO B e C são as que possuem as menores concentrações de $\mathrm{Cl}^{-}$(Tab. 1), o que pode ter contribuído para o resultado. De qualquer forma, é importante esclarecer que a elevação da $\mathrm{SID}_{3}$ foi pouco expressiva, e isso é coerente com o fato de que as soluções estudadas não causaram desequilíbrios eletrolíticos iatrogênicos. Ao contrário dos resultados do presente estudo, a ingestão da SEO B por bezerros sadios não provocou aumento da $\mathrm{SID}_{3}$ plasmática (Bachmann et al., 2009).

Por terem aumentado a $\mathrm{SID}_{3}$ do plasma, as SEO $\mathrm{B}$ e C provocaram efeito alcalinizante nos bezerros estudados. A alcalinização pode ser considerada leve porque as duas ingestões dessas SEO produziram incremento médio próximo de $4 \mathrm{mmol} / \mathrm{L}$ no BE, observado na hora 10,5 , não se tratando de modificação acentuada (Fig. 1). O $\mathrm{pH}$ sanguíneo foi ligeiramente aumentado e unicamente com a ingestão da SEO B. No estudo de Bachmann et al. (2009), os bezerros sadios que ingeriram a SEO $\mathrm{B}$ não apresentaram modificação do $\mathrm{pH}$ e da $\mathrm{pCO}_{2}$, o que contrasta com o resultado obtido. $\mathrm{O}$ equilíbrio ácido-base dos bezerros estudados não foi afetado pelas ingestões das SEO UEL, A e D e da SRL.

De acordo com a teoria dos íons fortes aplicada à interpretação do equilíbrio ácido-base, a SID efetiva de uma solução eletrolítica é a responsável decisiva pelo potencial que ela possui em causar ou não acidificação ou alcalinização (Smith e Berchtold, 2014). Soluções com SID efetiva elevada, maiores do que $40 \mathrm{mEq} / \mathrm{L}$, possuem teores elevados de $\mathrm{Na}^{+} \mathrm{e}$ reduzidos de $\mathrm{Cl}^{-}$, e provocam alcalinização diretamente proporcional ao valor da SID efetiva (Constable, 2003). Isso é comprovado em bezerros no caso das soluções de uso intravenoso (Junqueira et al., 2015), bem como no caso das SEO (Bachmann et al., 2009; 2012; Sayers et al., 2016). Teoricamente, portanto, as SEO A, B e D deveriam exercer efeitos alcalinizantes mais expressivos do que as SEO UEL e C, porque essas possuem valores de SID efetiva próximos ao valor da SID plasmática de bezerros sadios (Tab. 1) e, por isso, causariam menor impacto depois de absorvidas. Os resultados obtidos não confirmaram essa hipótese, e a provável explicação para isso é o fato de que as SEO estudadas possuíam valores de SID efetivas relativamente próximos. Cabe destacar que as SEO B e C são as que possuem as menores concentrações de $\mathrm{Cl}^{-}$, e essa pode ser a justificativa plausível para os seus efeitos alcalinizantes, distinguindo-as das outras.

Os efeitos ausentes ou pouco expressivos que as SEO estudadas provocaram sobre os equilíbrios eletrolítico e ácido-base dos bezerros podem ser justificados pelo fato de os animais serem hígidos. É possível admitir que os resultados poderiam ser diferentes caso as SEO fossem ingeridas por bezerros originalmente desidratados e desequilibrados. Essa hipótese é confirmada no estudo de Pagliosa et al. (2013), no qual a SEO C foi utilizada para tratar bezerros com diarreia induzida, portadores de desidratação e de acidose hiperclorêmica discreta. A ingestão dessa SEO produziu expansão do volume plasmático mais acentuada do que no presente estudo e alcalinização muito maior, gerando incremento médio do valor do BE próximo a $8 \mathrm{mmol} / \mathrm{L}$. Os resultados obtidos em bezerros sadios não podem ser, portanto, simplesmente extrapolados para os bezerros desidratados e acidóticos. O fato de a SEO A não ter produzido hemodiluição nos bezerros estudados não significa que ela seja provavelmente ineficaz para restabelecer o equilíbrio nos bezerros desidratados. Não se pode afirmar que as SEO B e C sejam as únicas indicadas para reverter a acidose metabólica. Pelo contrário, a avaliação dos efeitos das SEO estudadas em bezerros desidratados e a comparação entre as suas eficácias terapêuticas deverão ser investigadas em estudo controlado subsequente.

Os resultados do presente estudo possuem importância porque a hidratação não se aplica unicamente ao tratamento das diarreias e os bezerros desidratados por outros motivos podem não ter desequilíbrios eletrolíticos e ácido-base concomitantes. O uso das SEO estudadas pode ser considerado seguro nessas situações, exatamente por essas soluções não provocarem desequilíbrios iatrogênicos. Até mesmo nos 
quadros de diarreia em início da evolução, quando os desequilíbrios ainda são discretos, a ingestão das SEO estudadas pode ser admitida como medida eficaz porque pode prevenir o agravamento deles sem causar alcalose iatrogênica.

Por fim, o impacto das diferentes SEO estudadas sobre a glicemia dos bezerros sadios indica que a SEO D seria uma opção interessante como forma de minimizar a hipoglicemia nos casos em que o bezerro fosse privado de receber leite ou sucedâneo, pois sua alta concentração de glicose (109mmol/L) foi responsável por causar maior aumento na glicemia de bezerros sadios quando comparada com as demais SEO (Fig. 2). Pode-se supor que o mesmo efeito ocorreria nos animais hipoglicêmicos. Com a ingestão da SEO D, a hiperglicemia foi sustentada ao longo do dia, entre os picos de glicemia pós-prandiais. Curiosamente, o mesmo efeito não foi observado com a ingestão da SEO UEL, apesar de possuir concentração de glicose parecida com a da SEO D (Tab. 1). Outras características das composições dessas duas SEO não podem ser apontadas como explicação para a discrepância entre os resultados do efeito sobre a glicemia.

\section{CONCLUSÕES}

Conclui-se que todas as SEO utilizadas neste estudo são seguras para bezerros neonatos, pois não provocaram alterações intensas ou prolongadas nos equilíbrios hídrico, eletrolítico e ácido-base dos animais. A SEO A não foi capaz de expandir o volume plasmático, sinalizando baixo potencial terapêutico. As SEO B e C ocasionaram efeito alcalinizante, e, por gerar hiperglicemia duradoura, a SEO D deve ser usada com cautela em bezerros normoglicêmicos e não privados de leite ou de sucedâneo lácteo. Estudos controlados com bezerros desidratados e acidóticos são necessários para desafiar a eficácia terapêutica dessas soluções.

\section{AGRADECIMENTOS}

J.A.N. Lisbôa é bolsista de produtividade em pesquisa do $\mathrm{CNPq}$.

\section{REFERÊNCIAS}

BACHMANN, L.; HOMEIER, T.; ARLT, S. et al. Influence of different oral rehydration solutions on abomasal conditions and the acidbase status of suckling calves. J. Dairy Sci., v.92, p.1649-1659, 2009.

BACHMANN, L.; SCHMIDT, B.; RAUWOLF U. et al. Change of plasma volume, osmolality , and acid - base status in healthy calves after feeding of milk and water- and milk-based oral rehydration solutions. J. Dairy Sci., v.95, p.60066014, 2012.

CONSTABLE, P.D.; THOMAS, E.; BOISRAME, B. Comparison of two electrolyte solutions for the treatment of dehydrated calves with experimentally-induced diarrhoea. Vet. J., v.162, p.129-140, 2001.

CONSTABLE, P.D. Fluid and electrolyte therapy in ruminants. Vet. Clin. N. Am. Food Anim. Pract., v.19, p.557-597, 2003.

CONSTABLE, P.D.; GRUNBERG, W.; CARSTENSEN, L. Comparative effects of two oral rehydration solutions on milk clotting, abomasal luminal $\mathrm{pH}$, and abomasal emptying rate in suckling calves. J. Dairy Sci., v.92, p.296312, 2009.

COSENZA, M.; PEREIRA, P.F.V.; FERNANDES, L.L. et al. Efeito da solução de Ringer com lactato sobre os equilíbrios hidroeletrolíticos e ácido base de equinos, ovelhas e bezerros sadios. Cienc. Rural, v.43, p.2247-2253, 2013

JUNQUEIRA, J.R.C.; BALARIN, M.R.S.; FLAIBAN, K.K.M.C. et al. Efeito alcalinizante de soluções eletrolíticas com concentração elevada de lactato de sódio administradas em bezerros sadios. Arq. Bras. Med. Vet. Zootec., v.67, p.15-24, 2015.

NAYLOR, J.M. Oral fluid therapy in neonatal ruminants and swine. Vet. Clin. N. Am. Food Anim. Pract., v.6, p.51-67, 1990.

NOURI, M.; CONSTABLE, P.D. Comparison of two oral electrolyte solutions and route of administration on the abomasal emptying rate of Holstein-Friesian calves. J. Vet. Intern. Med., v.20, p.620-626, 2006. 
PAGLIOSA, G.M.; LIMA, A.K.S.; ENGEL, S. et al. Hidratação enteral associada ou não a antimicrobiano no tratamento de bezerros com diarreia experimentalmente induzida. Arq. Cienc. Vet. Zool. UNIPAR, v.16, p.113-120, 2013.

SAYERS, R.G.; KENNEDY, A.; KRUMP, L. et al. An observational study using blood gas analysis to assess neonatal calf diarrhea and subsequent recovery with a European Commission-compliant oral electrolyte solution. J. Dairy Sci., v.99, p.4647-4655, 2016.
SMITH, G.W. Treatment of calf diarrhea: oral fluid therapy. Vet. Clin. N. Am. Food Anim. Pract., v.25, p.55-72, 2009.

SMITH, G.W.; BERCHTOLD, J. Fluid therapy in calves fluid therapy calves diarrhea strong ion acidosis. Vet. Clin. N. Am. Food Anim. Pract., v.30, p.409-427, 2014.

VAN BEAUMONT, W.; GREENLEAF, J.E.; JUHOS, L. Disproportional changes in hematocrit, plasma volume, and proteins during exercise and bed rest. J. Appl. Physiol., v.33, p.55-61, 1972. 